Maternal Blood Group A and Pre-eclampsia

SIR,-We have been unable to substantiate Mr. D. P. L. May's findings (22 December, p. 738). An analysis of all primigravidae delivered of single pregnancies at St. Thomas's Hospital in 1969-70 (2,972 cases) has been carried out. In this study the diagnosis of pre-eclampsia was based on the findings at the last antenatal clinic. Mild pre-eclampsia was diagnosed when the diastolic blood pressure was $90 \mathrm{~mm} \mathrm{Hg}$ or more, with either proteinuria or oedema, and severe pre-eclampsia when the diastolic pressure was $100 \mathrm{~mm} \mathrm{Hg}$ or more, with proteinuria.

In our series 1,356 women $(45.6 \%)$ were of blood group $\mathrm{O}$ and $1,176(39 \cdot 6 \%)$ of group A. Dr. May found a very great increase in pre-eclampsia during the second half of pregnancy in women of group A compared with those of group $O$. In our cases there was very little difference between these two groups at term. The incidence of mild pre-eclampsia was $5 \cdot 10 \%$ (60 cases) in women of group A and $5.53 \%$ (75 cases) in those of group $O$. The corresponding figures for severe pre-eclampsia were $0.60 \%$ (7 cases) and $1 \cdot 18 \%$ (16 cases) respectively. These differences are not significant statistically.-We are, etc.,

JOANNA SOUTH JANET NALDRETT

Department of Gynaecology, St. Thomas's Hospital Medical School, London S.E.1

\section{Training for General Practice}

SIR,-I very much appreciated the letter from Dr. J. D. Sinson (23 February, p. 327). Qualifying from a British university does not ensure education in the specialty of general practice. Let us face the fact that at present the only hope of excellence in general practice is in the organization of postgraduate training in general practice. More and more doctors should receive inducement to study general practice. This is the only way in which new recruits to general practice can fight "the lack of morale bred by professional isolation or the temptation to freewheel and to channel the most difficult and intellectually-challenging cases to the hospitals" (Dr. F. M. Hull, Personal View, 23 February, p. 325). In fact this is what the creation of more chairs of general practice in Great Britain hopes to achieve. It is high time that the present occupants of general practice had a change of mind and attitude, and when selecting new recruits into general practice they should insist on their having completed a traineeship in general practice more than on anything else. -1 am, etc.,

Ruchill Hospital,

Glasgow

\section{Work Load in General Practice}

SIR,-With regard to the second article by Dr. G. N. Marsh and Mr. R. A. McNay (23 February, p. 319) we consider that it is important to note that their reference to our paper ${ }^{1}$ is inaocurate. They state that "like Whitfield we found that female patients who had recently joined the list did cause more work, though [Bain and Haines] have disagreed." If one reads our criticism of Whitfield's article ${ }^{2}$ it is evident that we were criticizing the method by which he had reached his conclusions, and as a result of the defects in methodology we were of the opinion that Whitfield's results could not be considered accurate or meaningful.

In addition, we would take issue with the statement by $\mathrm{Dr}$. Marsh and Mr. McNay in their first article in the same issue (p. 315) that "the average list size of 2,400 patients will rapidly become too small to occupy the time of established general practitioners. . . . For the majority an increase in list size will be mandatory to satiate their clinical interests." This statement cannot be acoepted on the basis of one general practitioner's experience. It may be that $\mathrm{Dr}$. Marsh cannot satisfy his clinical interests by looking after 3,000 patients, but he cannot assume that his methods of working are representative of all general practitioners in this country.-We are, etc.,

\section{J. G. BAIN}

A. J. HaINES

Craigshill Health Centre,
Livingston, West Lothian

1 Bain, D. J. G., and Haines, A. J., fournal of the Royal College of General Practitioners, 1973, 23, 73 . Whiffeld, M. J., Fournal of the Royal College of
General Practitioners, 1972, 22, 675.

\section{Perinatal Metabolism of Diazepam}

SIR,-We were interested to read the article by Dr. Jean E. Cree and others (3 November, p. 251) in which it was shown that diazepam and its main metabolite $\mathrm{N}$-demethyldiazepam were detectable in significant concentrations in the plasma of some infants for up to eight days postnatally if diazepam was given to the mothers less than 15 hours before delivery. We have found that diazepam and $N$-demethyldiazepam cross the placenta both in early ${ }^{1}$ and in late pregnancy. ${ }^{2}$ During subchronic use in early pregnancy diazepam accumulates in fetal tissues.' The newborn infant can also receive diazepam in the mother's milk if the mother is given diazepam post partum. ${ }^{4}$

To clarify the perinatal metabolism of diazepam we have examined the concentrations of diazepam and its metabolites in five newborn babies whose mothers received $10-15 \mathrm{mg}$ diazepam daily for 6-21 days before labour for the management of mild pre-eclampsia and sleep disturbances. The final dose was given 12-15 hours before delivery. Other treatment consisted of diuretics and antihypertensives. The concentrations of diazepam and its metabolites were determined by gas chromatography. ${ }^{2}$ The percentage recoveries of diazepam, $N$-demethyldiazepam, and free oxazepam zepam, $N$-demethyldiazepam, and free oxazepam were 95,85 , and 92 in both maternal and fetal
plasma. From the urine the percentage recoveries plasma. From the urine the percent
were 97,87 , and 96 , respectively.

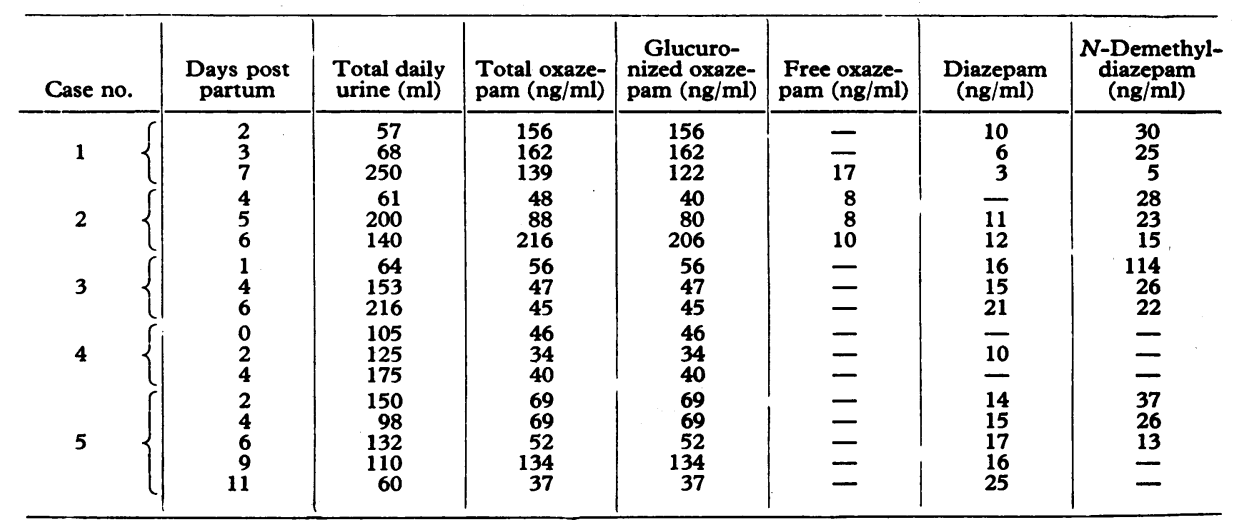

The concentrations of diazepam and $N$-demethyldiazepam in the babies' plasma are shown in the accompanying figure. The results are in good agreement with the results of Dr. Cree and her colleagues. The newborn infant seems to be ination takes at least 10 days. Free oxazepam was found in concentrations of $13-220 \mathrm{ng} / \mathrm{ml}$. One hin (case 4), hower, had the astonishingly high plasma free oxazepam concentration of $1,231 \mathrm{ng} / \mathrm{ml}$ immediately after delivery and this level persisted for three days post partum. In adults free oxazepam is found only in negligible concentrations. Our results give support to current views on the low glucuronizing capacity of the newborn with considerable individual variation.
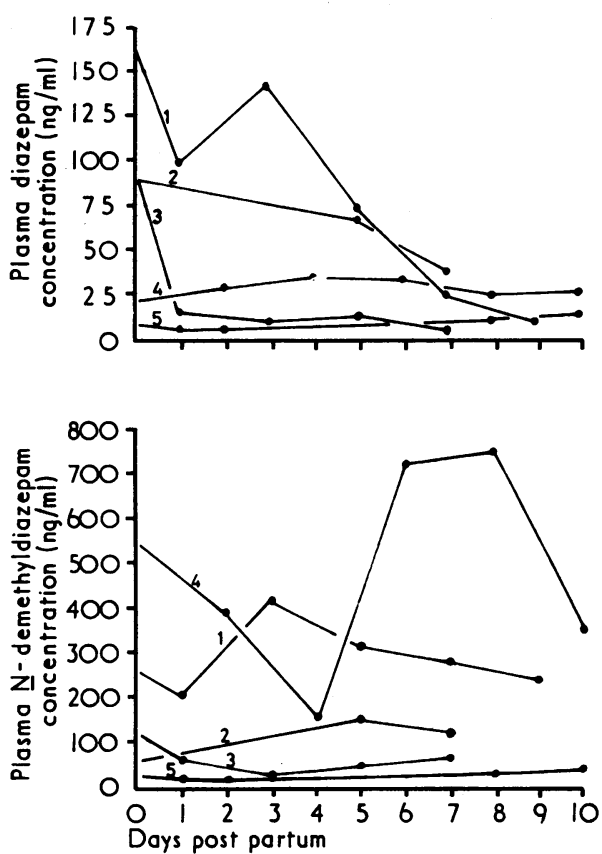

The concentrations of diazepam, $N$-demethyldiazepam, and oxazepam in the urine of the five infants are shown in the table. The main excreted component was glucuronized oxazepam, which constituted about $70 \%$ of all diazepam products in the urine. Glucuronized forms of diazepam and $N$-demethyldiazepam were not found. The infant who had the highest plasma concentration of free oxazepam (case 4) had the lowest total excretion of diazepam and its metabolites. individual variations in the metabolism and elimination of diazepam in the newborn. The concentrations of diazepam and its metabolites may be high enough to be pharmacologically active for up to 10 days. This can explain the respiratory difficulties, lethargy, and disturbances in thermoregulation to which Dr. Cree and her colleagues refer. Also the glucuronization of oxazepam may competitively inhibit the conjugation of bilirubin, leading to hypercapable of metabolizing diazepam, but its eliminfant (case 4), however, had the astonishingly

Our results indicate that there are wide 
bilirubinemia in the newborn, as has been suggested. 5 Finally, practically nothing is known about the influence of diazepam upon the developing fetal brain or the developing neurological functions of the newborn. Thus we do not recommend the subchronic use of diazepam in any stage of pregnancy or in doses higher than 10-20 mg to the mother during labour.-We are, etc.,

J. KANTO R. ERKKOLA R. SELLMAN

Departments of Pharmacology and of Obstetrics and Gynaecology,

Turku, Finland

1 Erkkola, R., Kanto, J., and Sellman. R., Excerpta Medica Intemational Conference Series 1973, $279,109$.

2 Erkkola, R., Kangas, L., and Pekkarinen, A., Acta Obstetricia et Gynecologica Scandinavica, 1973, 52, 167.

3 Erkkola, R., and Kanto, J., in Third European Congress of Perinatal Medicine, ed. H. Bossart, p. 310. Berne, Huber, 1972.

I Erkkola, R., and Kanto, J., Lancet, 1972, 1, 1235. Rosanelli, K., Geburtshilfe und Frauenheilkunde,
1970, 30, 713.

\section{Adverse Reactions to Intra-amniotic Urea and Prostaglandin}

SIR,-Since July 1973 it has been our practice to induce mid-trimester therapeutic abortion by the technique of intra-amniotic urea and prostaglandin $E_{2}$ injection after amniocentesis, as described by Craft. ${ }^{1}$ In the main we have found this method satisfactory, with a minimum of adverse affects. However, we here describe three cases in which adverse reactions, all of a similar nature, occurred at the time of induction of abortion.

The patients, who were 14-16 weeks pregnant, received no premedication and all passed urine within 30 minutes of the start of the procedure. The adverse reaction common to all three cases consisted of rigors, vomiting, severe abdominal pain, and an intense desire to pass urine and defaecate. There was also peripheral vasoconstriction and a rapid, low-volume pulse, with hypotension in one case and peripheral cyanosis in another. In two cases, in which the liquor was clear, the lower abdominal pain followed immediately after lower abdominal pain followed immediately after the injection of the urea solution and preceded the injection of $5 \mathrm{mg}$ of prostaglandin. In the third
case, in which the liquor was blood-stained, no urea was injected and only $2 \mathrm{mg}$ of prostaglandin by slow injection was used. The first two cases responded, with a return to normal within on hour, to a rapid intravenous infusion of normal saline and intravenous hydrocortisone, diazepam, and promethazine; abortion subsequently occurred. The third patient required an infusion and curred. The third patient required an infusion and was induced two weeks later by intra-amniotic was induced two weeks later by
hypertonic saline without incident.

The effects of large doses of intravenous prostaglandin are well known, and the features exhibited by the patients described could well represent the effects of a massive dose of prostaglandin introduced into the systemic circulation. This could possibly explain the phenomena in the case in which blood-stained liquor was obtained and one would advise against the use of prostaglandins or urea in such circumstances. However, in the two cases in which the liquor was clear it was more difficult to ascribe the reactions to this cause. These were the cases in which the injection of urea solution caused severe lower abdominal pain. One would therefore advise against the use of prostaglandins when the injection of the urea solution causes lower abdominal pain even though clear liquor was obtained at amniocentesis.
As this series of events has not to our knowledge been previously recorded, we would appreciate any details of similar cases.-We are, etc.

ALAN H. ROSS

Queen Mary's Hospital,

W. L. WHITEHOUSE

1 Craft, J. L., Lancet, 1973, 1, 1344.

\section{CCNU in Treatment of Recurrent Medulloblastoma}

SIR,-CCNU is 1-(2-chloroethyl)-3-cyclohexyl-1-nitrosourea and is one of the nitrosoureas synthesized at the U.S. National Cancer Institute. It has an advantage over 1,3-bis (2-ahloroethyl)-3-cyclohexyl-1- nitrosourea (BCNU) in that it can be given by mouth and has a higher lipid solubility. The latter property is probably an important reason why the drug can cross the bloodbrain barrier effectively. ${ }^{1}$ CCNU has been reported to produce marked improvement in the condition of patients with glioblastoma multiforme ${ }^{2}$ and glioma. ${ }^{3}$ Hence it seemed reasonable to assess its value in children with recurrent medulloblastoma.

A 7-year-old boy presented in September 1971 with vomiting, headache, nystagmus, papilloedema, and ataxia. Craniotomy and biopsy showed a differentiating medulloblastoma in the cerebellum. Megavoltage radiotherapy was given to the whole central nervous system, the spinal cord receiving 4,000 rads and the brain between 4,600 and 5,400 rads over six weeks, the highest dosage being in the posterior fossa. Subsequently his condition the posterior fossa. Subsequently his condition In December 1972 he developed metastases in the in December 1972 he developed metastases in the biopsy. These were irradiated. Intermittent treatment with cyclophosphamide and vincristine was instituted but later vinblastine was substituted because vincristine caused severe constipation. There was complete relief of pain and the child returned to school. In August 1973, despite perseverance with the above chemotherapy, he developed right facial and ocular muscular palsies. veloped right facial and ocular muscular palsies.
On 4 August $1973100 \mathrm{mg}$ of CCNU $\left(130 \mathrm{mg} / \mathrm{m}^{8}\right.$ On 4 August $1973100 \mathrm{mg}$ of CCNU (130 mg/ms
body surface area) was given. Three weeks later body surface area) was given. Three weeks later weeks after starting treatment both ocular and acial palsies were barely detectable. Treatment with CCNU was repeated once every six weeks. The child remained well three months after starting treatment, with no depression of the blood count or abnormality of liver function tests.

A 9-year-old girl presented in April 1971 with vomiting, headache, nystagmus, right lateral rectus palsy, and ataxia. Craniotomy and biopsy showed a medulloblastoma in the vermis. Megavoltage radiotherapy was given to the whole voltage radiotherapy was given to the whole central nervous system, the spinal cord receiving 4,000 rads and the brain 4,500 rads over five weeks.
Subsequently her condition returned to normal Subsequently her condition returned to normal apart from slight past-pointing with the left hand.
In May 1972 she developed dysphagia and weakness of the legs. Further megavoltage radiotherapy was given to the posterior fossa, amounting to 2,500 rads over five days, and intermittent treatment with cyclophosphamide and vincristine was started. A month later she was remarkably well and no neurological abnormality could be found. and no neurological abnormality could be found. In March 1973 she complained of cramp and slight weakness in the legs, so vinblastine was substituted was less. On 1 September she suddenly developed severe headache and vomiting and two weeks late she was moribund, with quadriplegia, complete dysphagia, and inability to speak. CCNU 120 mg was given via a nasogastric tube. Ten days later she was able to talk, 'swallow, and walk with assistance. Treatment with CCNU was repeated assistance. Treatment with CCNU was repeated
six weeks later. Two months after starting treatsix weeks later. Two months after starting treat-
ment she remained 'well apart from occasional lack ment she remained 'well apart from occasional lack blood count or abnormality of liver function tests.

Both these patients showed a remarkable degree of recovery after treatment with CCNU despite the fact that severe deteriora- tion in their condition had occurred while they were on treatment with cyclophosphamide and vinblastine. This suggests that CCNU is a valuable drug for the treatment of medulloblastoma, though this does not preclude the addition of other drugs such as one of the vinca alkaloids. Furthermore, the simplicity of treatment by a single oral dose every six weeks must favour its use after radiotherapy in the planned initial treatment of medulloblastoma as well as for recurrent disease.

I thank Dr. M. Slavik and the U.S. National Cancer Institute for supplying the CCNU used in this trial.

-I am, etc.

Department of Radiotherapy,

H. W. C. WARD

Queen Elizabeth Hospital,

Birmingham

1 Rall, D. P., and Zubrod, C. G., Annual Review of Pharmacology, 1962, 2, 109. Hansen, H. H., Selawry, O. S.. Muggia, F. M.,
and Walker. M. D., Cancer Research, 197i, 31, 223.

Walker, M. D., Rosenblum, M. L., Smith, K. A., and Reynolds, A. F., jun., Proceedings of the American Association for Cancer Research,

\section{Levodopa and Chronic Bronchitis}

SIR,-The beneficial effect of levodopa noted by Dr. H. G. Jeffs ( 9 March, p. 454) has been observed previously, ${ }^{1}$ though it is by no means invariable. ${ }^{2}$ One possible mechanism to account for such a bronchodilator action might be through generation of the Sohiff base derivative tetrahydropapaveroline, ${ }^{3}$ which is known to be a $\beta$-adrenergic agonist. ${ }^{+}-\mathrm{I}$ am, etc.,

MERTON SANDLER

Queen Charlotte's Maternity Hospital,

1 Ingvarsson, G., Nordisk Medicin, 1965, 74,

1166.
Fleming, P., Makar, H., and Hunter, K. R. Lancet, 1970, 2, 1186.

Sandler, $M$., Bonham Carter, S., Hunter, K. R., Holtz, P., Stock, K., Nature, 1973, 241, 439. Nounyn-Schmiedeberg's Archiv für experiNounyn-Schmiedeberg's Archiv für experi-
mentelle Pathologie und Pharmakologie, 1964,

SIR, $\rightarrow$ Dr. H. G. Jeffs's interesting report (9 March, p. 454) of improvement in dyspnoea apparently due to levodopa is consistent with the results of a double-blind study on pulmonary function in patients with Parkinson's disease which showed a significant increase in expiratory flow rates during treatment with levodopa.1 This was attributed to improvements in motivation and akinesia, but bronchiolar dilation is an attractive additional explanation. However, when levodopa was given to an asthmatic with a druginduced extrapyramidal syndrome there was a greatly increased frequency of attacks of bronchial asthma.2 Clearly, further studies on the actions of levopoda in obstructive airways disease are required. Perhaps other factors such as metabolic effects of levopoda are important in patients with abnormal bronchial secretions.-I am, etc.

K. R. HUNTBR

Bristol Royal Infirmary,

1 Nakano, K. R., Bass, H., and Tyler, H. R., Archives of Intermal Medicine, 1972, 130, 346. Lancet, 1970, 2, 1186. 